\title{
In vitro gas production provides effective method for assessing ruminant feeds
}

\author{
Girma Getachew \\ Edward J. DePeters \\ Peter H. Robinson \\ $\nabla$
}

An animal's feed intake, and how well that feed is digested, determine the feed's production performance. The in vitro gas production technique is a relatively simple method for evaluating feeds, as large numbers of samples can be incubated and analyzed at the same time. This method has been applied successfully at UC Davis for a variety of purposes in feed evaluation, including calculating organic matter digestibility, the metabolizable energy of feeds and kinetics of their fermentation; determining how feed value is affected by added fat, antinutritive factors and rumen modifiers; quantifying the energy value of feed mixtures (rations); monitoring microbial change in the rumen; synchronizing nutrient digestion; and selecting forage nutrient targets for agricultural biotechnology. More than half of the nutrients consumed by ruminant animals leave the animal unutilized and undigested, and are excreted in feces, urine and gases. The in vitro gas production method can be used to examine animal waste components that impact the environment and develop appropriate mitigations.

$\mathrm{R}$ uminants have a four-compartment stomach. The rumen is the largest compartment, where millions of bacteria grow under anaerobic (low-oxygen) conditions. These bacteria are responsible for the digestion of fiber (cellulose) and are the reason why ruminants can

To test the digestibility of animal feeds, UC Davis graduate students Kelly McCaughey and Stefanie Cheng help postdoctoral associate Girma Getachew collect rumen fluid from "Tank." The cow has been fitted with a permanent fistula, allowing daily collection of rumen fluid.

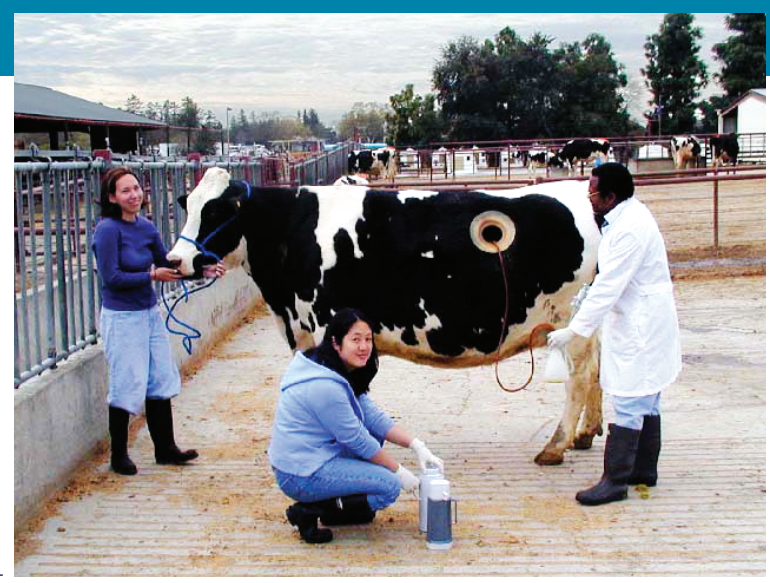

consume a wide variety of byproduct feedstuffs derived from the processing of plants for human food. California livestock industries utilize the majority of these highly fibrous byproducts by including them in feeds for cattle, sheep and goats. The nutritive value, or energy content, of an animal feed is determined predominately by its digestibility, which affects intake, or how much the animal will eat.

Digestibility and intake, in turn, determine the feed's productive performance, such as to support milk synthesis or muscle growth. However, studies with live animals (in vivo) to determine the digestibility of feeds are time-consuming, laborious, expensive and require large quantities of feed. Such experiments are not suited for the rapid and routine feed evaluations undertaken by commercial laboratories that provide feed information to livestock producers and feed manufacturers.

The digestibility of feeds can also be estimated by biological methods known as in vitro techniques, which are conducted outside of the animal system but simulate the digestion process. Generally, in vitro techniques are those based on measuring either fermentation residues or products. The former measures the unfermented residue remaining after in vitro incubation of a feed with rumen fluid. This approach involves collecting fluid by hand from the rumen of a ruminant that has been fitted with a rumen fistula (see photo above). This method for forage evaluation was first reported in 1963, using ruminal fluid obtained from a sheep with a rumen fistula (Tilley and Terry 1963). A rumen fistula is formed by surgically transecting the skin and the rumen, suturing the rumen to the skin and allowing the rumen to heal, creating a permanent opening into the rumen. A soft cannula fits in the fistula to close the rumen. The cannula can be opened to allow access to the rumen in order to collect the bacteria-containing fluid needed for in vitro incubations. Material not recovered in the residue following incubation is assumed to be fermented, providing estimates of the extent of digestion for various feeds.

More recent methods measure the products of anaerobic fermentation. Rumen fermentation by anaerobic microbes results in production of shortchain fatty acids (SCFA), gases (carbon dioxide $\left[\mathrm{CO}_{2}\right]$ and methane $\left.\left[\mathrm{CH}_{4}\right]\right)$ and microbial mass. The amount of gas produced is proportional to acid production, thereby serving as an indicator of acids produced by fermentation. The amount of gas produced during incubation is measured to predict the extent and rate of feed digestion.

In addition to quantifying the chemical composition of feeds, some commercial laboratories offer in vitro feed digestibility as a component of their feed analysis packages. This data can be used in new ration-evaluation computer models with the goal of optimizing nu- 


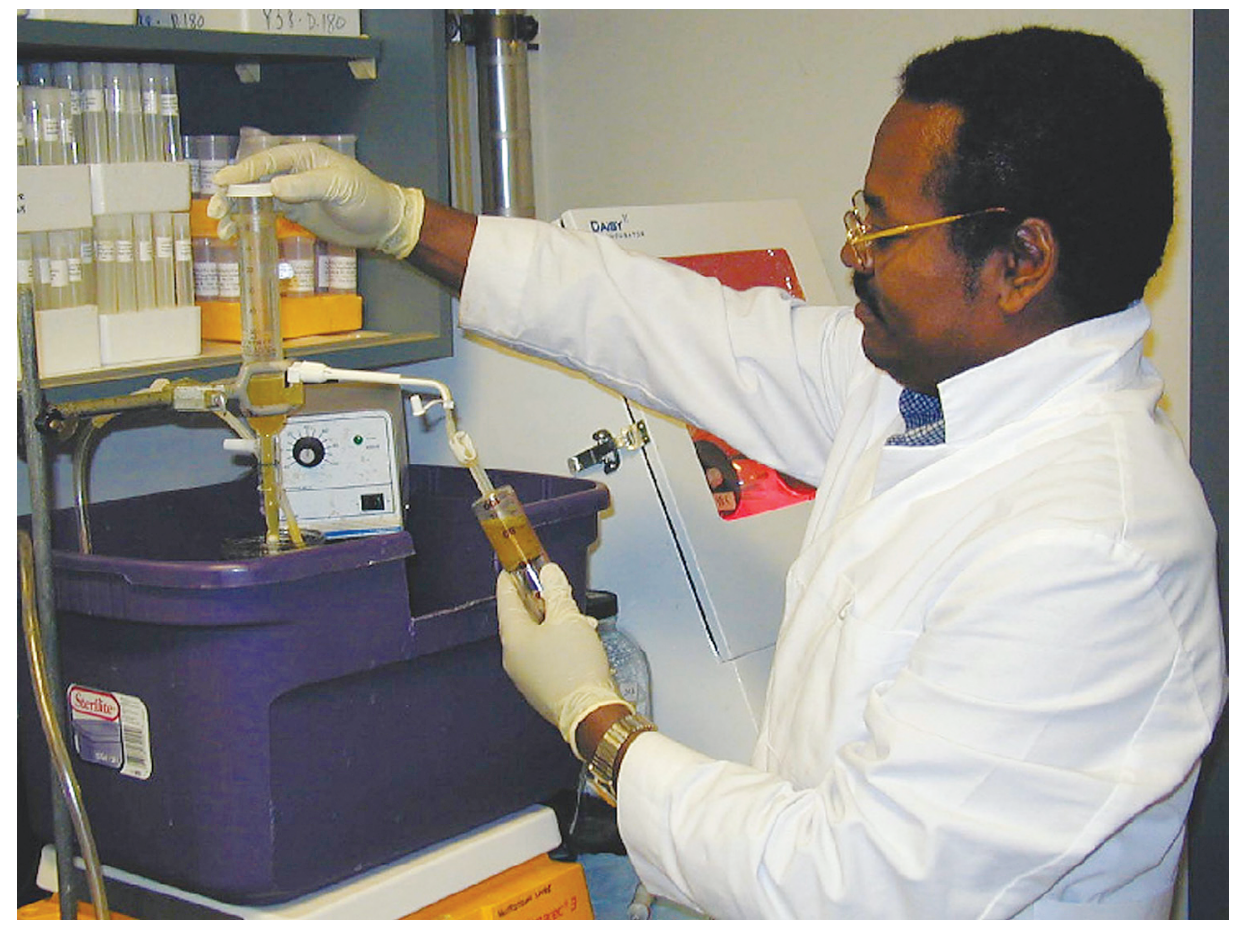

trient utilization and animal productive performance, thereby minimizing the environmental impacts of nutrient excretion in the animal's urine and feces.

Gas-measuring techniques have been routine in feed evaluation since the early 1980s, when a high correlation was found between metabolizable energy (ME) measured in live animals and that predicted from gas production. The in vitro gas technique has several advantages over other in vitro methods that are based on measuring residues. Gas production reflects all nutrients fermented, soluble as well as insoluble; and fractions that are not fermentable do not contribute to gas production. Furthermore, the kinetics of fermentation can be obtained from a single incubation, allowing the rate of fermentation to be calculated.

Gas measurement is a direct measure of microbial activity and can be a better index of forage ME content than an indirect in vitro measure based on nutrients fermented. The gas technique is relatively simple and does not require sophisticated equipment, making it easy to conduct for research and commercial purposes. Rumen fluid is collected from a cow with a rumen fistula. Fermentations are conducted in large (100 milliliter [ml]) calibrated glass syringes in an anaerobic medium inoculated with rumen fluid. Incubations can be carried out either in an incubator with a rotating disc or in a thermostatically controlled water bath $\left(102^{\circ} \mathrm{F}\right)$. The vol- ume of gas produced in 24 hours from incubating 200 milligrams (mg) of feed, together with the concentration of crude protein and crude fat, is used to predict ME. Large numbers of samples can be analyzed during a single 24-hour incubation run.

At UC Davis, three nonlactating Holstein cows (about 1,450 pounds each) are fitted with permanent rumen fistulas. Twice daily, the cows are fed a diet of mostly oat hay with a small amount of alfalfa hay. This ensures that the ruminal microbial population changes little from day to day. Rumen fluid is collected after the morning feeding using a manually operated vacuum pump. The fluid is placed into prewarmed thermos flasks, then mixed and filtered through four layers of cheesecloth and flushed with carbon dioxide in the laboratory. One part rumen fluid is mixed with two parts buffered mineral solution (1:2 volume/volume) and maintained at $102^{\circ} \mathrm{F}$. Finally, this buffered rumen fluid $(30 \mathrm{ml})$ is pipetted into incubation syringes containing the ground test substrate and placed in a $102^{\circ} \mathrm{F}$ water bath. Gas production is measured by visually reading the scale on the side of each syringe.

\section{Applicability of gas method}

The in vitro gas method has been applied successfully at UC Davis for a variety of purposes in feed evaluation.

Organic matter digestibility. The digestibility of measured organic matter
Compared with laborious and expensive in vivo testing, the in vitro gas production method provides a quick and easy way to calculate organic matter digestibility, quantify the energy value of feed mixtures and monitor microbial change in the rumen. Left, Girma Getachew fills syringes with buffered rumen fluid.

is closely correlated with that predicted from gas production and the crude protein and ash contents of feeds. Therefore, the method can be used to predict the extent of digestion for various feeds.

Energy contents of feeds. The gas method has also been used successfully to predict the ME content of feeds. A regression equation has been developed with data generated by in vivo studies conducted with a variety of feeds and in vitro gas production. The gas measurement provides a better estimate of the ME level of feeds, when combined with some chemical constituents, compared with calculations based on chemical constituents only. Recently, seven laboratories around the world that use a gas method - including UC Davis - carried out a comparative test to assess the repeatability of the technique in predicting the energy value of feeds, and found that the gas method was repeatable among laboratories (Getachew et al. 2002).

Kinetics of fermentation. In assessing nutritive value, the rate at which a feed or its chemical constituents are digested in the rumen is as important as the extent of digestion. The pattern of feed fermentation (kinetics of fermentation) is one of several factors that influence voluntary feed intake by ruminants. The rate at which different chemical constituents are fermented is a reflection of microbial growth and accessibility of the feed to microbial enzymes. By describing gas produc- 
tion mathematically, kinetic data can be analyzed to evaluate substrate- and media-related differences as well as the fermentability of soluble and slowly fermentable components of feeds. The gas method is an ideal technique to generate kinetics of fermentation, as it allows recording of gas produced at several times in the incubation period, which is used to predict the rate at which feed is digested.

The gas method has been used to evaluate the effects of grain processing on the rate and extent of gas production (DePeters et al. 2003)(fig. 1). Steamflaked corn fermented faster than whole grain. The first 16 hours of incubation were important for measuring starch availability. Different types of forages, including cereal hays and silages, were also studied to determine their patterns of fermentation in the rumen. Although alfalfa hay fermented faster during the early stages, it was lower in extent of gas production compared to corn silage after 72 hours of

incubation (fig. 2).

Effects of added fat on feed degradation. Tallow and yellow grease (YG), both rendering byproducts, are typical fats used in the diets of lactating dairy cows. The gas technique was used at UC Davis to examine the effect of sources and levels of added fat on gas production and rumen fermentation of a total mixed ration (Getachew et al. 2001)(fig. 3). Fatty acids in the form of triglyceride (YG) had no effect (when

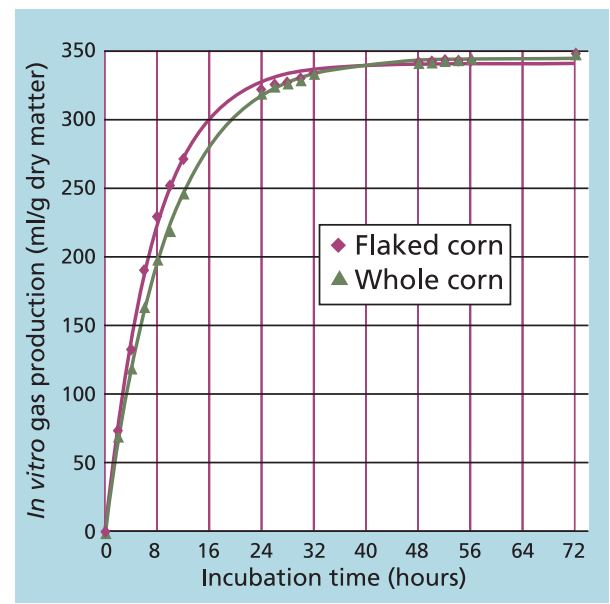

Fig. 1. Kinetics of in vitro gas production during incubation of flaked and whole corn grain.

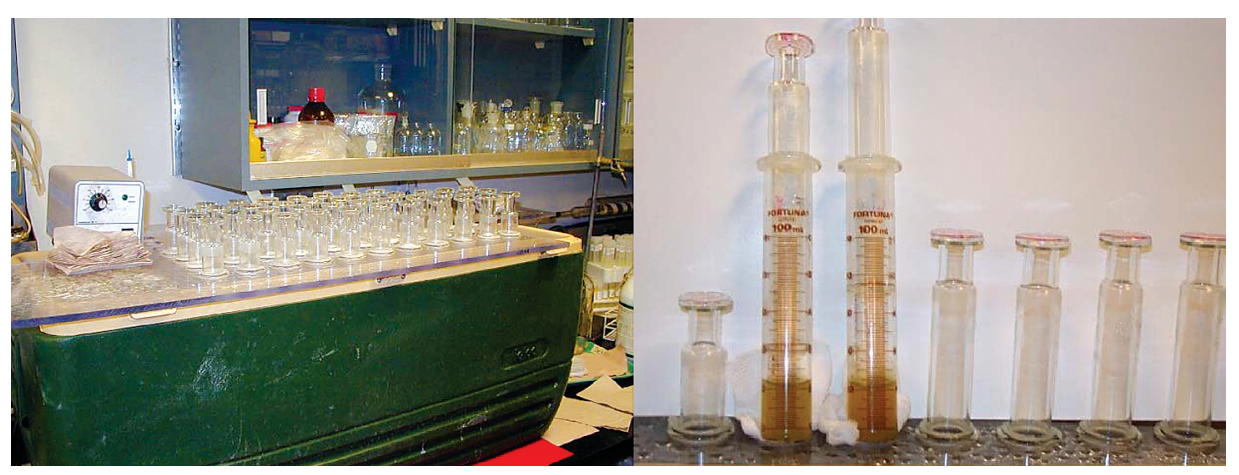

Left, dozens of syringes containing feed samples can be incubated at the same time during a single 24-hour incubation run. Right, gas production is measured by reading the scale on the side of the syringe.

comprising up to $25 \%$ of the diet) on gas production, but fatty acids in the form of potassium salts (YG soap) significantly depressed gas production. In the animal, however, there is a limit to the amount of fatty acids that can be successfully fed, and this is lower than in vitro. The fatty acids in potassium salts are quickly available to microbes as free fatty acids in ruminal fluid, and have detrimental effects on microbial growth. In contrast, the fatty acids in the triglyceride form must be released through hydrolysis of the ester bond and therefore are available at a slower rate. Hydrolysis refers to breaking the chemical bond between the individual fatty acid and the glycerol backbone of the triglyceride. The effects of fatty acids on rumen fermentation are important because feeds with high levels of residual fat, for example rice bran created in the production of white rice, are commonly fed to ruminants.

Antinutritive factors. The gas method can be used to measure how micro-

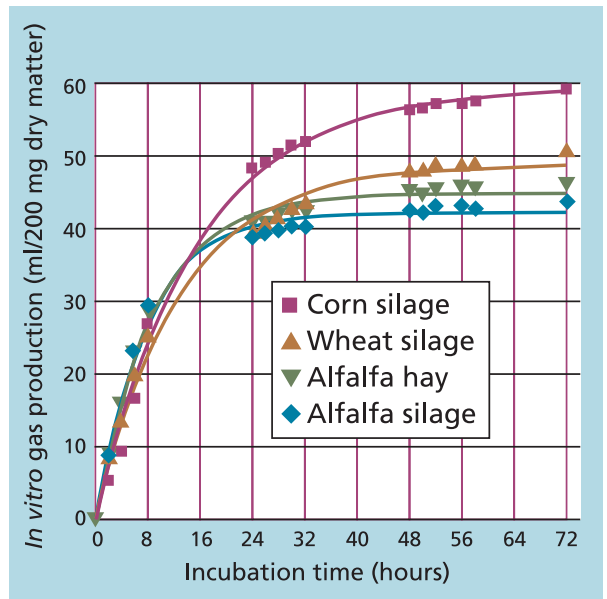

Fig. 2. Pattern of in vitro gas production during incubation of alfalfa hay and alfalfa, corn and wheat silages. bial activity lowers feed digestibility. Some feeds, such as forage legumes and cottonseed, contain phenolics, alkaloids and saponins that have negative biological effects on microbes and reduce microbial growth in rumen. Tannins are naturally occurring polyphenolic compounds found in plants, which form complexes with feed and microbial proteins and can depress feed digestibility in the rumen. The effect of tannins on the nutritive value of feeds can be studied using tannin-binding agents, such as polyethylene glycol (PEG), which strongly binds to tannins and inhibits their biological effects. The percent increase in gas production when PEG is present indicates the rate at which tannins depress rumen fermentation of feeds.

After adding PEG to limit tannin effects, gas production increased by $22 \%, 71 \%$ and $211 \%$ in apple ring acacia (Acacia albida), beach acacia (Acacia cyanophylla) and red calliandra (Calliandra calothyrsus), respectively, which

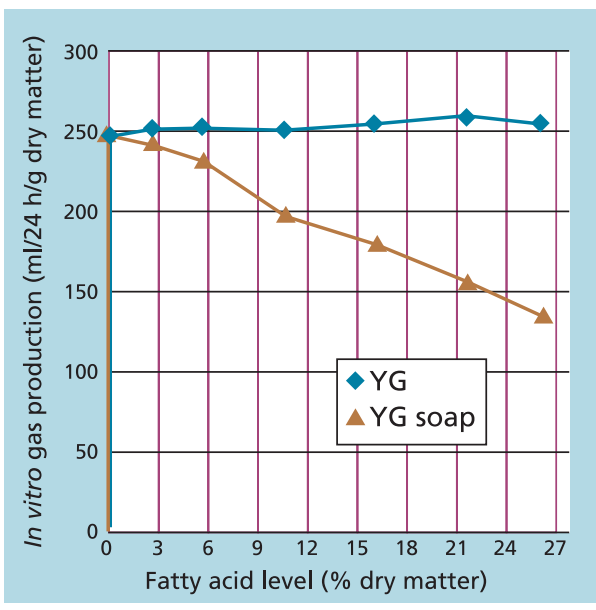

Fig. 3. Effect of yellow grease (YG) and yellow grease soap (YG soap) on in vitro gas production. 
are browse plants (Getachew et al. 2000b)(fig. 4).

Rumen modifiers. The gas method is also utilized to study feed additives and rumen fermentation modifiers, such as monensin sodium, by incubating feeds in the presence or absence of these compounds. Rumen modifiers are compounds that are added to the diet to modify the populations of bacteria in the rumen. For example, some compounds are fed to reduce methanogenic bacteria to reduce methane production in the rumen. Previous studies have shown that the addition of saponins and tannins in an in vitro system increases microbial protein synthesis (Makkar et al. 1995). Yeast and yeast fermentation products are routinely added to the diets of lactating dairy cows, although their mode of action has not been clearly identified. By studying the impact of various rumen modifiers on microbial fermentation, effects important to milk production in commercial dairy farms can be quantified.

Feed associative effects. The in vitro gas production method is currently being used to assess "associative" effects of feeds used in rations. Rations are mixtures of individual feeds, with a multitude of possible combinations. The energy value of a ration is generally calculated by adding up the energy values of the individual feeds in the ration, on the assumption that the individual energy value of any particular feed is the same in every possible combination with other feeds. However, this is not always true. For example, when poorquality forage - such as wheat straw -

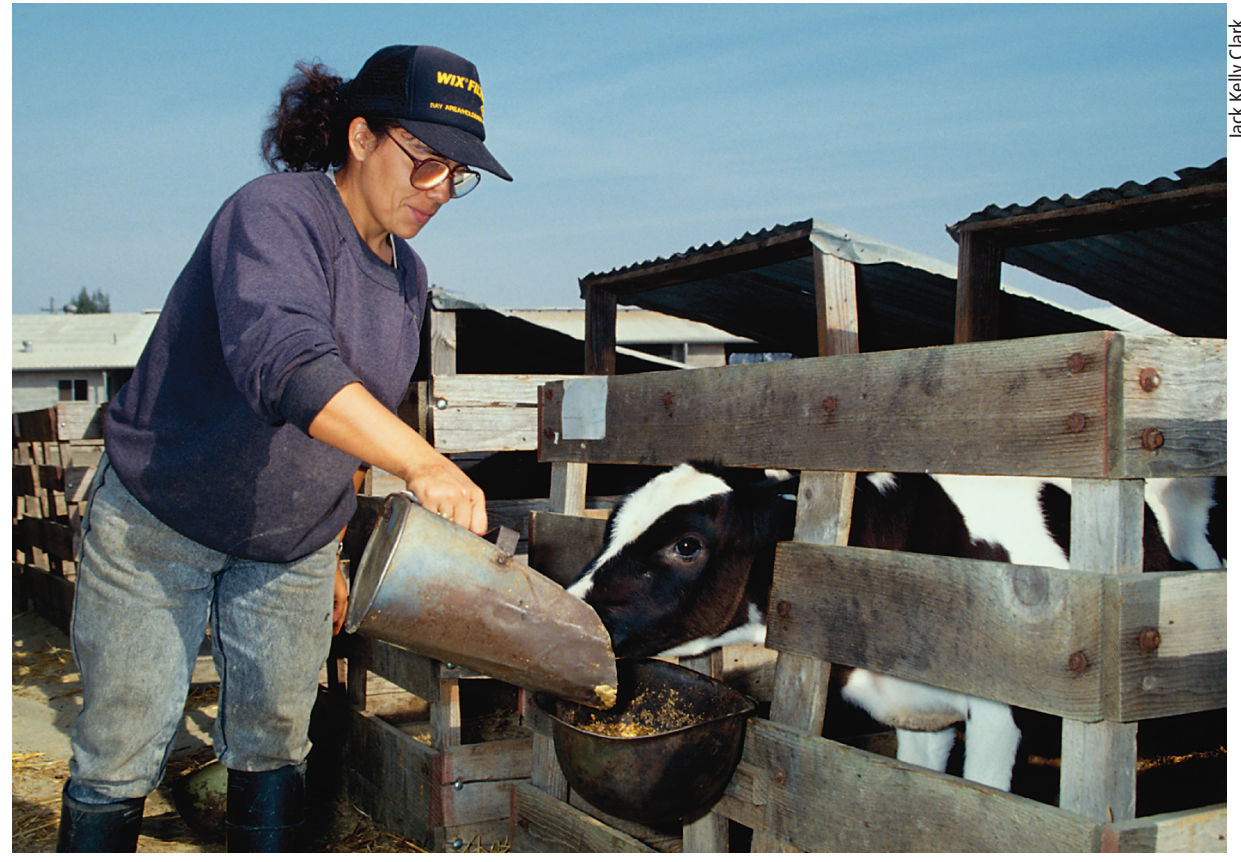

More than half of the nutrients consumed by ruminant animals such as cows are undigested and excreted into the environment. By using feed evaluations to improve feed utilization, air and water pollution can be reduced. is fed to a ruminant, its digestibility is low, but by adding nitrogen in the form of urea or protein, the digestibility of the straw will be increased and in turn, the energy derived from straw organic matter in the diet will be increased. Recent studies indicate that positive associative effects on in vitro gas production occurred when rice straw was incubated in mixtures with hay or mulberry leaves (Liu et al. 2002).

\section{Monitoring rumen microbial}

change. In addition to rates and extents of digestion, the gas production method can be used to study substraterelated factors that influence microbial manipulation of rumen microflora to increase the utilization of feeds through populations in the rumen. This enables

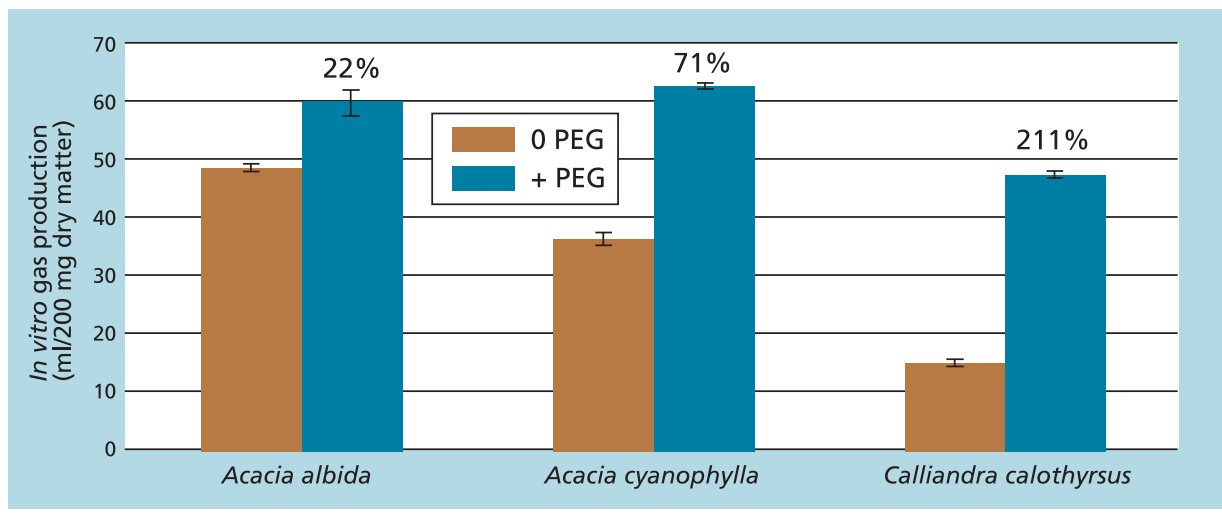

Fig. 4. In vitro gas production of tannin-containing leaves in absence of PEG (0 PEG, with tannins) and presence of PEG (+ PEG, with no tannins). degradation of fiber and lignin, increasing the efficiency of nitrogen utilization or allowing the degradation of antinutritional and toxic components of feeds. Such controlled fermentation systems could potentially be used with genetic engineering of plants to solve animal productivity problems. The technique is suitable for application of molecular-based assays, such as polymerase chain reaction (PCR) and ribonucleic acid (RNA)-targeted oligonucleotide probes, to study and measure rumen microbial growth, with the goal of increasing the efficient utilization of feeds and reducing environmental impacts. Recently, Muetzel and Becker (2003) used the gas technique, in combination with ribosomal RNAtargeted probes, to measure the efficiency of microbial growth when barley straw was supplemented with legume leaves.

Nutrient synchronization. Carbohydrate and nitrogen sources must be available simultaneously in order to maximize microbial growth. Ruminal ammonia concentrations can be influenced by the degradation rates of carbohydrates and nitrogen-containing compounds. For a given level of dietary protein, an increased rate of protein degradation enhances the ruminal ammonia concentration while an increased rate of carbohydrate degradation decreases it. 
Increased carbohydrate availability for fermentation promotes microbial growth and as a result less nitrogen is lost from the rumen in the form of ammonia-nitrogen (Getachew et al. 2000a). The gas method offers an opportunity to study microbial requirements for nitrogen and carbohydrate to enable efficient fermentative activity and accumulation in the rumen. Using this technique, studies have been conducted to assess rumen microbial requirements for nitrogen when different types of carbohydrate sources are incubated.

Plant breeding, biotechnology. We believe that animal nutritionists and plant geneticists should collaborate to select genetic materials that have better agronomic performance and superior nutritional qualities. Siaw et al. (1993) used the gas technique to evaluate large numbers of browse species in order to select those high in feeding value. Browse is the edible parts of woody vegetation such as leaves, stems and twigs from bushes common on California foothills; they have been identified as integral to the development of fires that ravaged Southern California in the fall of 2003. Several forage and cereal crops have been genetically modified to increase yield, or produce chemical constituents normally deficient in a particular plant. Forage plants are selected for rapid fiber digestibility. Plants have also been genetically engineered to produce human lysozyme, but it is unclear what effect lysozyme has on microbes in the rumen. Although many genetically engineered plants are intended for human consumption, their byproducts will be fed to animals as a means of disposal. The starch contained in cereals, including corn and milo, is found in granules surrounded by a tough protein matrix that reduces enzymatic degradation. There are new genetic varieties of these cereals with modified protein matrices. We are currently using the gas production method to explore whether these new varieties increase the extent and rate of starch digestion (DePeters et al. 2003).

Environmental degradation. More than half of the nutrients consumed by ruminants leave the animal unutilized and undigested, and are excreted in feces, urine and gases. This increases animal production costs as well as environmental impacts, by contaminating surface- and groundwater and contributing to air pollution. The nitrogenous and organic compounds excreted are further decomposed and can cause odors in residential areas. Increasing the efficiency of feed utilization reduces the amount of unutilized nutrients leaving the animal. Significant reductions in nitrogenous compounds (Kuelling et al. 2003) and in methane (Johnson and Johnson 1995) can be achieved by manipulating animal diets. The in vitro gas method can be used to study the efficiency of feed utilization and to examine animal waste components that impact the environment in order to develop appropriate mitigation strategies.

\section{Determining nutritive value}

The nutrient composition of feeds is commonly determined primarily by chemical analyses. However, this does not provide sufficient information to determine the feed's true nutritive value. The efficiency by which an animal utilizes feed nutrients has a significant impact on its productive performance and waste production. The in vitro gas production system helps to better quantify nutrient utilization, and its accuracy in describing digestibility in animals has been validated in numerous experiments. Animal experiments will continue to add information to our understanding of nutrient metabolism. However, where applicable the in vitro gas production system can be used to predict animal performance at a much lower cost. Based on the strong relationship between measured digestibility and that predicted from gas production, regression equations have been developed and the method has been standardized.

In addition, the method can evaluate the impact of biotechnological changes in plants on their nutritive value and other factors that affect rumen fermentation.
G. Getachew is Postdoctoral Associate, E.J. DePeters is Professor, and P.H. Robinson is Cooperative Extension Specialist, Department of Animal Science, UC Davis. This research was supported by the California Agricultural Experiment Station, UC Davis.

\section{References}

DePeters EJ, Getachew G, Fadel JG, et al. 2003. In vitro rumen gas production as a method to compare fermentation characteristics of steam-flaked corn. Animal Feed Sci Technol 105:109-22.

Getachew G, Crovetto GM, Fondevila M, et al. 2002. Laboratory variation of $24 \mathrm{~h}$ in vitro gas production and estimated metabolizable energy value of ruminant feeds. Animal Feed Sci Technol 102:169-80.

Getachew G, DePeters EJ, Robinson PH, Taylor SJ. 2001. In vitro rumen fermentation and gas production: Influence of yellow grease, tallow, corn oil and their potassium soaps. Animal Feed Sci Technol 93:1-15.

Getachew G, Makkar HPS, Becker K. 2000a. Effect of polyethylene glycol on in vitro degradability of nitrogen and microbial protein synthesis from tannin-rich browse and herbaceous legumes. Br J Nutr 84:73-83.

Getachew G, Makkar HPS, Becker K. 2000b. Tannins in tropical browses: Effects on in vitro microbial fermentation and microbial protein synthesis in media containing different amounts of nitrogen. J Agric Food Chem 48:3581-8.

Johnson KA, Johnson DE. 1995. Methane emissions from cattle. J Animal Sci 73:248392.

Kuelling DR, Menzi H, Sutter F, et al. 2003. Ammonia, nitrous oxide and methane emissions from differently stored dairy manure derived from grass- and hay-based rations. Nutrient Cycling Agroecosystems 65:13-22.

Liu JX, Susenbeth A, Sudekum KH. 2002. In vitro gas production measurements to evaluate interactions between untreated and chemically treated rice straw, grass and mulberry leaves. J Animal Sci 80:517-24.

Makkar HPS, Blümmel M, Becker K. 1995. In vitro effects of and interactions between tannins and saponins and fate of tannins in the rumen. J Sci Food Agric 69:481-93.

Muetzel S, Becker K. 2003. Supplementation of barley straw with Sesbania pachycarpa leaves in vitro: Effects of fermentation variables and rumen microbial population structure quantified by ribosomal RNA-targeted probes. Br J Nutr 89:445-53.

Siaw DEKA, Osuji PO, Nsahlai IV. 1993. Evaluation of multipurpose tree germplasm: The use of gas production and rumen degradation characteristics. J Agric Sci (Cambridge) 120:319-30.

Tilley JM, Terry RA. 1963. A two-stage technique for the in vitro digestion of forage crops. J Br Grassland Soc 18:104-11. 